$02,13,12$

\title{
Моделирование критического состояния слоистых сверхпроводящих структур с неоднородными слоями
}

\author{
(С) П.И. Безотосный, К.А. Дмитриева \\ Физический институт им. П.Н. Лебедева РАН, \\ Москва, Россия \\ E-mail: bezpi@lebedev.ru
}

Поступила в Редакцию 26 мая 2021 г.

В окончательной редакции 26 мая 2021 г.

Принята к публикации 3 июня 2021 г.

\begin{abstract}
Предложен подход к расчету критического состояния слоистых структур, состоящих из неоднородных сверхпроводящих слоев. Метод основан на численном решении одномерных уравнений Гинзбурга-Ландау, обобщенных для неоднородной пластины. Метод позволяет получить зависимости критического тока от магнитного поля, а также распределение тока и магнитного поля по слоям. Проведено сравнение усредненного критического тока слоистых структур, состоящих как из неоднородных, так и из однородных слоев. Получено, что при относительно небольшом количестве слоев и малой величине внешнего магнитного поля критический ток слоистых структур с однородными слоями может превосходить критический ток структур с неоднородными слоями. С увеличением количества слоев и/или величины внешнего магнитного поля критический ток слоистых структур с неоднородными слоями начинает, наоборот, превышать критический ток структур с однородными слоями. Сила пиннинга в структурах с неоднородными слоями выше, чем в случае с однородными.
\end{abstract}

Ключевые слова: сверхпроводящие слоистые структуры, сверхпроводящие пленки, критический ток, теория Гинзбурга-Ландау, неоднородность.

DOI: 10.21883/FTT.2021.10.51393.125

\section{1. Введение}

Исследования свойств реальных структур из сверхпроводящих материалов не теряют своей актуальности [1-4]. Одним из основных методов моделирования таких структур является теория Гинзбурга-Ландау (ГЛ) [1-3]. Интерес представляют моделирование критического тока в структурах с пиннингом [5-7], зарождение вихрей Абрикосова в сверхпроводниках второго рода и структурах из этих сверхпроводников $[3,8,9]$.

Базовым элементом многих сверхпроводящих структур являются тонкие пленки. Корректное описание свойств сверхпроводящих пленок требует учета их особенностей, к которым могут быть отнесены, в том числе, существенное влияние границ пленок на их свойства [10-12] и неоднородность по толщине [13-16]. Развитие моделей, описывающих свойства тонких сверхпроводящих пластин, позволяет улучшить оценки параметров структур, составной частью которых они являются. Например, в работе [17] учет влияния границ отдельных слоев на критическое состояние слоистых структур позволяет приблизить оценки критического тока и силы пиннинга к наблюдаемым на практике.

В настоящей статье предложен метод расчета критического состояния слоистых структур, состоящих из неоднородных сверхпроводящих слоев. Метод позволяет получить зависимости усредненного по слоям критического тока от магнитного поля, а также распределение тока и магнитного поля по слоям. В работе проведено сравнение усредненного критического тока слоистых структур из неоднородных слоев со слоистыми структурами из однородных слоев. Для каждой из структур проведены расчеты зависимостей силы пиннинга от внешнего магнитного поля.

\section{2. Описание модели}

В работе рассматривается слоистая структура, представляющая собой набор длинных и широких сверхпроводящих пластин (слоев) толщины $D$ (длина и ширина пластин много больше $D$ ) в магнитном поле $\mathbf{H}$, параллельном поверхности пластин. В каждой из пластин течет транспортный ток, перпендикулярный внешнему магнитному полю. В качестве транспортного тока $I_{t}$ используется произведение его плотности на толщину пластины, т.е. ток, приходящийся на единицу ширины пластины. Используется декартова система координат $(x, y, z)$, при этом оси $y$ и $z$ направлены параллельно поверхности слоев. Внешнее магнитное поле сонаправлено оси $z$, а транспортный ток - оси $y$ (см. рис. 1). В данной геометрии векторный потенциал имеет вид $\mathbf{A}=\mathbf{e}_{y} A(x)$.

Предполагается, что сверхпроводящие свойства каждой отдельной платины изменяются от центра к границам. В связи с этим, неоднородность сверхпроводящих пластин задается через зависимость длины свободного 


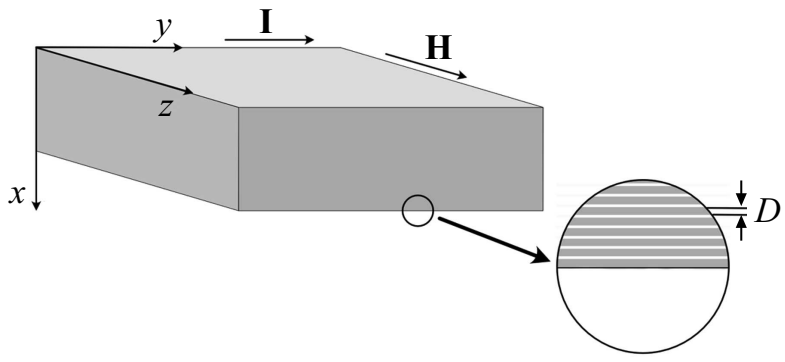

Рис. 1. Геометрия задачи.

пробега от координаты х следующим образом:

$$
l(x)=l_{C n}\left(1-\eta\left(\frac{x}{D}-\frac{1}{2}\right)^{2}\right),
$$

где $l_{C n}$ - длина свободного пробега в центре пластины, а $\eta$ - параметр, характеризующий степень ее неоднородности. Случай однородной пластины соответствует $\eta=0$, при этом $l(x)=l_{C n}$.

Для выбранной зависимости $l(x)$ уравнения ГЛ для отдельной пластины принимают вид (в работах [14-16] подробно описан вывод уравнений):

$$
\begin{aligned}
\psi-\psi^{3} & +\left(1-\eta\left(\frac{x_{\xi}}{d}-\frac{1}{2}\right)^{2}\right) \frac{\partial^{2} \psi}{\partial x_{\xi}^{2}}-\frac{2 \eta}{d}\left(\frac{x_{\xi}}{d}-\frac{1}{2}\right) \frac{\partial \psi}{\partial x_{\xi}} \\
& -\frac{U^{2}}{\kappa_{C n}^{2}} \psi\left(1-\eta\left(\frac{x_{\xi}}{d}-\frac{1}{2}\right)^{2}\right)=0 \\
& \frac{\partial^{2} U}{\partial x_{\xi}^{2}}-\frac{\psi^{2}}{\kappa_{C n}^{2}} U\left(1-\eta\left(\frac{x_{\xi}}{d}-\frac{1}{2}\right)^{2}\right)=0
\end{aligned}
$$

где $\kappa_{C n}-$ параметр ГЛ в центре пластины, $\psi-$ нормированный параметр порядка: $\psi=\Psi / \sqrt{a / 2 b}, a$ и $b-$ коэффициенты, $\Psi$ - параметр порядка в разложении функционала свободной энергии $F$ (см. [14-16]). Вместо размерных значений координаты $x$, толщины пластины $D$, потенциала $A$ и плотности тока в сверхпроводнике $j_{s}$ здесь введены безразмерные величины $x_{\xi}, d, U\left(x_{\xi}\right)$, и $j\left(x_{\xi}\right)$ соответственно:

$$
\begin{gathered}
x_{\xi}=x / \xi_{C n}, \quad d=D / \xi_{C n}, \quad U=\frac{2 \pi \kappa_{C n} \xi_{C n}}{\phi_{0}} A, \\
j=\frac{8 \pi^{2} \kappa_{C n}^{3} \xi_{C n}^{3}}{c \phi_{0}} j_{s}, \quad \xi_{C n}=\sqrt{f_{C n} / a},
\end{gathered}
$$

где $\phi_{0}-$ квант магнитного потока, $\xi_{C n}-$ длина когерентности ГЛ однородного сверхпроводника или длина когерентности ГЛ в центре неоднородного сверхпроводника.

Для решения уравнения (2) используются следующие граничные условия:

$$
\begin{aligned}
& \left.\frac{d \psi}{d x_{\xi}}\right|_{x_{\xi}=0}=0, \\
& \left.\frac{d \psi}{d x_{\xi}}\right|_{x_{\xi}=d}=0 .
\end{aligned}
$$

Транспортный ток $I_{t}$ проходящий через $i$-ую пластину, создает магнитное поле:

$$
H_{I}=\frac{2 \pi}{c} I_{t}
$$

Полное магнитное поле вблизи поверхностей пластины равно $H \pm H_{I}$, и граничные условия для уравнения (3) имеют вид:

$$
\left.\frac{\partial U}{\partial x}\right|_{x_{\xi}=0}=h-h_{I},\left.\quad \frac{\partial U}{\partial x}\right|_{x_{\xi}=d}=h+h_{I},
$$

где

$$
h=\frac{H}{H_{\xi}}, \quad h_{I}=\frac{H_{I}}{H_{\xi}}, \quad H_{\xi}=\frac{\phi_{0}}{2 \pi \kappa_{C n}^{2} \xi_{C n}^{2}} .
$$

Входящие в уравнения ГЛ глубина проникновения магнитного поля $\lambda$ и длина когерентности $\xi$ зависят от температуры, в связи с чем полученные выражения являются неявными функциями температуры.

Задача нахождения критического тока слоистой структуры состоит из двух этапов. Сначала на основе самосогласованного решения системы обобщенных уравнений ГЛ $(2,3)$ находится зависимость критического тока $I_{c}$ от величины внешнего магнитного поля $H$ для отдельной пластины. При этом полагается, что сверхпроводящие пластины находятся в безвихревом состоянии. Итерационная процедура решения системы уравнений $(2,3)$ описана в работе [12]. Стоит отметить, что подход к моделированию критического состояния отдельного слоя, используемый на первом этапе, аналогичен подходу для случая отдельной неоднородной пластины [14-16].

На втором этапе определяется критический ток слоистой структуры. При этом предполагается, что джозефсоновским взаимодействием между слоями можно пренебречь. Это обусловлено тем, что плотность джозефсоновского тока меньше плотности тока распаривания, поэтому она мало влияет на амплитуду параметра порядка в слоях и на распределение транспортного тока по ним. Для учета электрического взаимодействия между сверхпроводящими слоями делается допущение, что все они соединены сверхпроводящими перемычками при $y= \pm \infty$. При этом целью поиска является такое распределение транспортного тока по слоям, при котором все слои переходят в нормальное состояние одновременно. Если $h_{i}$ - магнитное поле, в котором находится $i$-ый слой, то в критическом состоянии через него пропускается ток на единицу ширины пленки, равный критическому току пластины $I_{c}\left(h_{i}\right)$, который определяется на основе численного решения уравнений ГЛ в первой части сформулированной задачи. Тогда во всех слоях структуры устанавливается критическая плотность тока.

Согласно принципу суперпозиции для нахождения магнитного поля, в котором находится произвольный 
$i$-ый сверхпроводящий слой, необходимо просуммировать вклады каждого слоя

$$
h_{i}=h+\sum_{j=1}^{i-1} h_{t j}-\sum_{j=i+1}^{N} h_{t j},
$$

где $h_{t j}$ - безразмерное магнитное поле, создаваемое транспортным током, протекающим через $j$-ый слой. Распределения критического тока и магнитного поля по слоям, при котором все слои переходят в нормальное состояние одновременно, находится методом последовательных приближений $[18,19]$.

Все используемые далее значения длины и толщины представлены в единицах длины когерентности в центре пластины $\xi_{C n}$, а значения магнитного поля - в единицах $H_{\xi}$, что упрощает сравнение свойств слоистых структур из неоднородных и однородных пластин (в случае последних $\eta=0$ ). Значения силы тока в рамках модели представляются через $H_{I}(4,5)$ и, по аналогии с магнитным полем, выражены в единицах $H_{\xi}$.

В работе обсуждаются результаты моделирования проведенного при следующих параметрах слоистых структур: толщина слоев $d=2$, степень их неоднородности $\eta=3$ (задается в распределении длины свободного пробега электронов $l$ по толщине (1)), параметр ГЛ в центре слоя $\kappa_{C n}=10$. Рассматриваются слоистые структуры, состоящие из $N=1,100,200$ и 500 слоев. Результаты расчетов сравниваются с результатами, полученными для слоистых структур, состоящих из однородных слоев.

\section{3. Результаты численных расчетов}

Для обсуждения полученных результатов введем усредненный по слоям структуры критический ток $\left\langle I_{c}\right\rangle$ в виде

$$
\left\langle I_{c}\right\rangle=\frac{1}{N} \sum_{i=1}^{N} I_{c i},
$$

где $N$ - количество слоев в слоистой структуре. Значения $\left\langle I_{c}\right\rangle$ приводятся в безразмерных единицах. Случай $N=1$ соответствует отдельной пластине, критическое состояние которой моделировалось в работах [14-16].

Зависимости $\left\langle I_{c}\right\rangle$ от внешнего магнитного поля для слоистых структур с количеством слоев $N=1$ и 200 представлены на рис. 2,a. Изменение зависимостей $\left\langle I_{c}\right\rangle(h)$ при учете неоднородности отдельных слоев по толщине для случая относительно небольшого количества слоев $(N=200)$ аналогично изменению данной зависимости для отдельной пластины $(N=1)$. Критический ток слоистой структуры из неоднородных слоев в отсутствие внешнего магнитного поля ощутимо уменьшается по отношению к критическому току слоистой структуры из однородных слоев. В свою очередь критическое магнитное поле в отсутствие транспортного тока существенно возрастает для структуры из неоднородных слоев. С увеличением количества слоев $(N=500)$
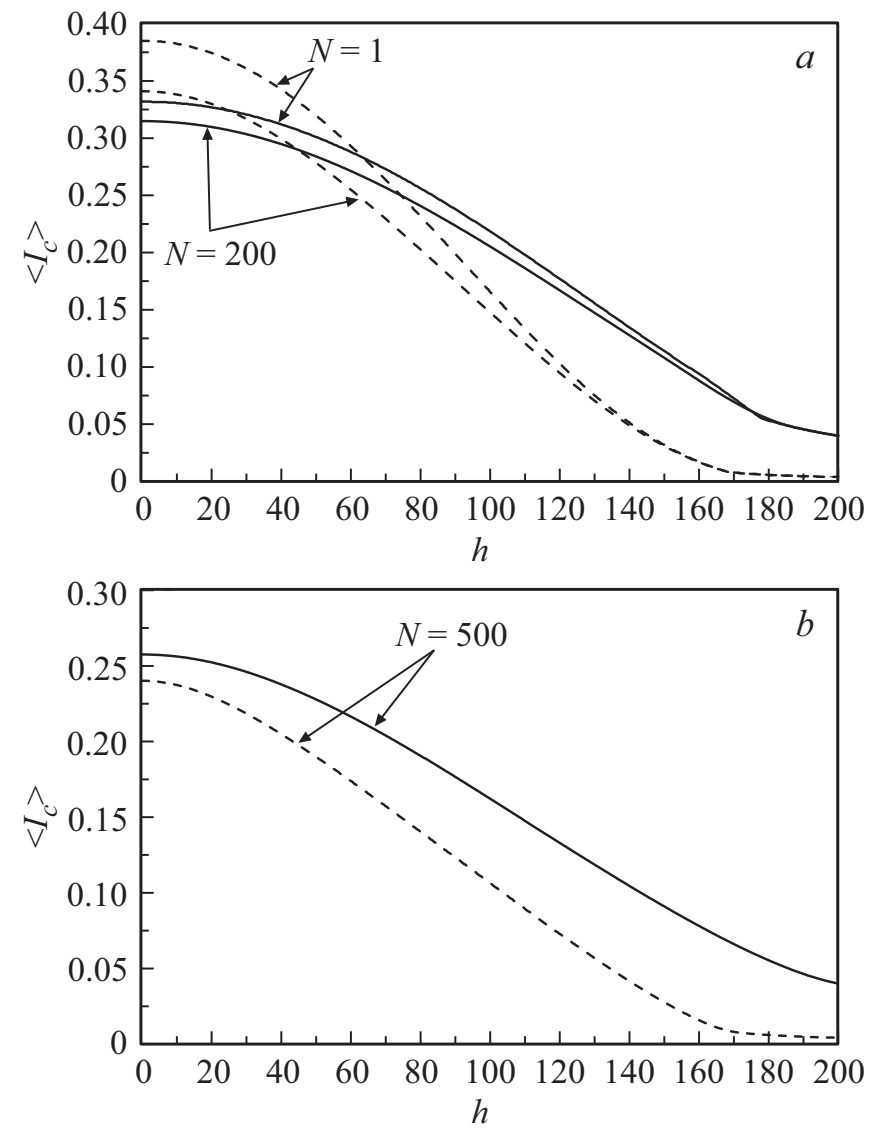

Рис. 2. $(a-b)-$ - Зависимости плотности критического тока от внешнего магнитного поля $\left\langle I_{c}\right\rangle(h)$ для слоистых структур с разным количеством слоев $(N)$ (их количество указано около соответствующих кривых) $(a)-N=1$ и 100; $(b)-$ $N=500$. Зависимости представлены для случаев однородных слоев (выделены пунктирной линией), и неоднородных слоев (выделены сплошной линией). Предполагается, что все слои имеют толщину $d=2, \kappa_{C n}=10\left(\kappa_{C n}-\right.$ параметр ГЛ в центре неоднородной пластины или параметр ГЛ однородной пластины). В случае неоднородных слоев степень неоднородности $\eta=3$.

усредненный по неоднородным слоям критический ток начинает превосходить критический ток структуры из однородных слоев при всех величинах внешнего магнитного поля, в том числе, в его отсутствие (см. рис. $2, b$ ). Это связано с тем, что при увеличении количества слоев в структуре создаваемое транспортным током магнитное поле существенно увеличивается (в силу суперпозиции полей отдельных слоев), и каждый отдельный слой находится в большем по величине магнитном поле. Критический ток отдельного неоднородного слоя, начиная с некоторой величины внешнего магнитного поля, начинает превосходить критический ток отдельного однородного слоя (график, соответствующий $N=1$ на рис. 2,a). Если магнитное поле распределено по структуре таким образом, что большая часть слоев находится в магнитном поле, при котором критический ток неоднородного слоя больше критического тока однородного 


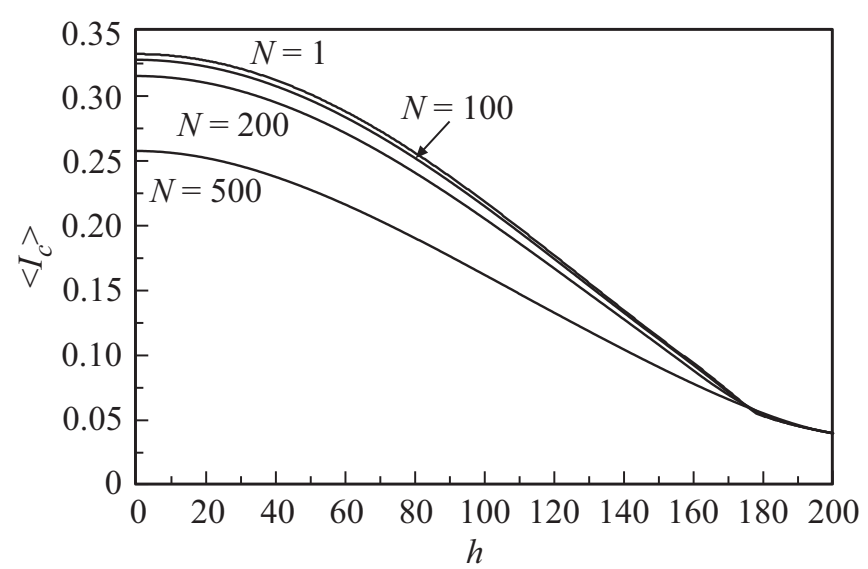

Pис. 3. Зависимости плотности критического тока от внешнего магнитного поля $\left\langle I_{c}\right\rangle(h)$ для слоистых структур с разным количеством слоев $(N)$ (их количество указано около соответствующих кривых). Зависимости представлены для случая неоднородных слоев со степенью неоднородности $\eta=3$. Предполагается, что все слои имеют толщину $d=2, \kappa_{C n}=10$ $\left(\kappa_{C n}-\right.$ параметр ГЛ в центре пластины).
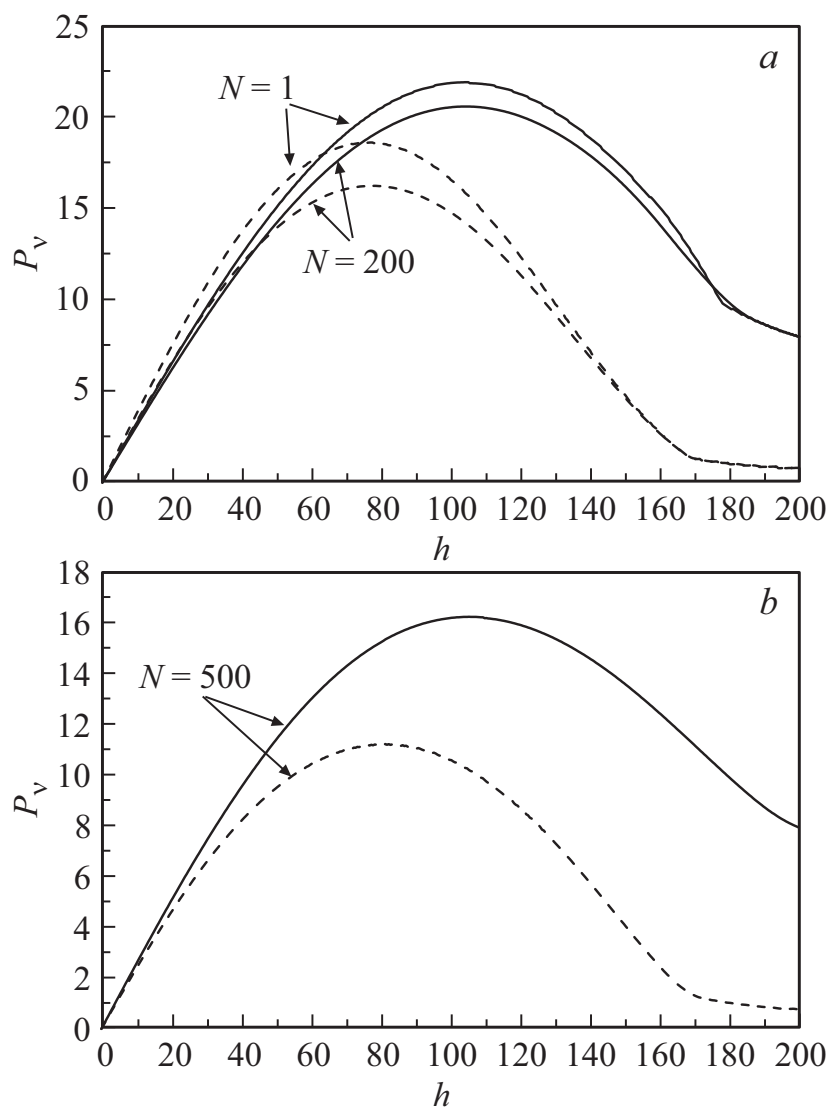

Pис. 4. $(a-b)-$ Зависимости объемной силы пиннинга от внешнего магнитного поля $P_{v}(h)$ для слоистых структур с разным количеством слоев $(N)$ (их количество указано около соответствующих кривых) $(a)-N=1$ и $100 ;(b)-N=500$. Зависимости представлены для случаев однородных слоев (выделены пунктирной линией), и неоднородных слоев (выделены сплошной линией). Предполагается, что все слои имеют толщину $d=2, \kappa_{C n}=10\left(\kappa_{C n}-\right.$ параметр ГЛ в центре неоднородной пластины или параметр ГЛ однородной пластины). В случае неоднородных слоев степень неоднородности $\eta=3$.

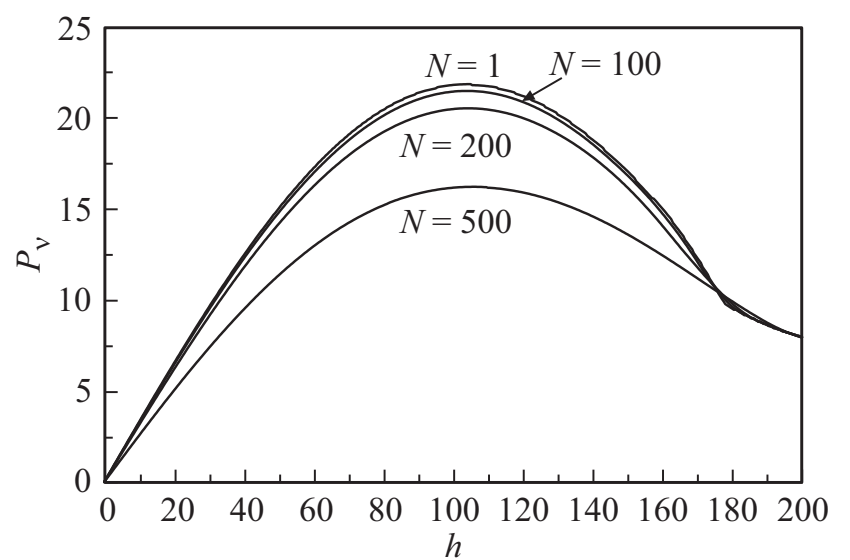

Рис. 5. Зависимости объемной силы пиннинга от внешнего магнитного поля $P_{v}(h)$ для слоистых структур с разным количеством слоев $(N)$ (их количество указано около соответствующих кривых). Зависимости представлены для случая неоднородных слоев со степень неоднородности $\eta=3$. Предполагается, что все слои имеют толщину $d=2, \kappa_{C n}=10\left(\kappa_{C n}\right.$ параметр ГЛ в центре пластины).

слоя, то и усредненный критический ток структуры из неоднородных слоев начинает превышать критический ток структуры из однородных слоев.

Изменение зависимостей $\left\langle I_{c}\right\rangle(h)$ с увеличением $N$ для случая слоистых структур из неоднородных слоев (см. рис. 3) аналогично изменению данных зависимостей для структур из однородных слоев [17]. С увеличением количества слоев увеличивается магнитное поле, которое они создают. Это приводит к уменьшению плотности критического тока. С увеличением внешнего магнитного поля влияние слоев уменьшается, и усредненный критический ток приближается к $I_{c}(h)$ для одного слоя.

Подобно описанному в работе [17] рассмотрим аналог объемной силы пиннинга: $P_{v}(h)=\left\langle I_{c}\right\rangle h$. На рис. $4(a-b)$ показаны примеры зависимости объемной силы пиннинга от внешнего магнитного поля. Результаты приведены для случаев структур из неоднородных (зависимости выделены сплошными линиями) и однородных слоев (зависимости выделены сплошными линиями). На рис. 4, $a$ приведены результаты расчетов для слоистых структур с количеством слоев $N=1$ и 200 , а на рис. $4, b$ с количеством слоев $N=500$. Качественно изменение объемной силы пиннинга при учете неоднородности слоев по толщине схоже для слоистых структур с различным количеством слоев и представляет собой увеличение максимума силы пиннинга и сдвиг его в сторону более сильных полей. Таким образом при одинаковых значениях $\mathrm{N}$ сила пиннинга структур из неоднородных слоев превосходит силу пиннинга для структур с однородными слоями.

Изменение зависимостей $P_{v}(h)$ с увеличением $N$ для случая слоистых структур из неоднородных слоев (см. рис. 5) аналогично изменению данных зависимостей для структур из однородных слоев [17]: с увеличением количества слоев объемная сила пиннинга уменьшается. 


\section{4. Заключение}

Основные результаты работы могут быть сформулированы следующим образом:

- предложен подход к моделированию критического состояния слоистых структур, учитывающий неоднородность их слоев по толщине;

- сравнение усредненного критического тока слоистых структур из неоднородных слоев со слоистыми структурами из однородных слоев показывает, что если количество слоев и величина внешнего магнитного поля относительно небольшие, то критический ток слоистых структур с однородными слоями превосходит критический ток структур с неоднородными слоями, что также наблюдается в случае отдельной пластины. С увеличением количества слоев и/или величины внешнего магнитного поля критический ток слоистых структур с неоднородными слоями начинает превышать критический ток структур с однородными слоями. В случае структуры с достаточно большим количеством слоев критический ток неоднородной структуры больше, чем у однородной при всех величинах внешнего магнитного поля, в том числе, при его отсутствии. Стоит отметить, что для отдельной пластины подобное поведение не наблюдается.

- сила пиннинга больше в структурах с неоднородными слоями, что также характерно для отдельной пластины.

Разработанный метод моделирования критического состояния слоистых структур может быть полезен при анализе свойств реальных композитных сверхпроводников.

\section{Финансирование работы}

Работы выполнена в рамках Государственного задания Министерства науки и высшего образования РФ (тема „Физика высокотемпературных сверхпроводников и новых квантовых материалов“, № 0023-2019-0005).

\section{Конфликт интересов}

Авторы заявляют, что у них нет конфликта интересов.

\section{Список литературы}

[1] D.Yu. Vodolazov, G. Berdiyorov, F.M. Peeters. Physica C 552, 64 (2018).

[2] D.Yu. Vodolazov, Yu.P. Korneeva, A.V. Semenov, A.A. Korneev, G.N. Goltsman. Phys. Rev. B 92, 10, 104503 (2015).

[3] Alden R. Pack, Jared Carlson, Spencer Wadsworth, Mark K. Transtrum. Phys. Rev. B 101, 14, 144504 (2020).

[4] Yu.P. Korneeva, D.Yu. Vodolazov, A.V. Semenov, I.N. Florya, N. Simonov, E. Baeva, A.A. Korneev, G.N. Goltsman, T.M. Klapwijk. Phys. Rev. Appl. 9, 6, 064037 (2018).

[5] A.N. Moroz, A.N. Maksimova, V.A. Kashurnikov, I.A. Rudnev. IEEE Transact. Appl. Supercond. 28, 4, 8000705 (2018).

[6] V.A. Kashurnikov, A.N. Maksimova, A.N. Moroz, I.A. Rudnev. Supercond. Sci. Technol. 31, 11, 115003 (2018).
[7] В.А. Кашурников, А.Н. Максимова, И.А. Руднев, Д.С. Одинцов. ФТТ 57, 9, 1685 (2015).

[8] K.S. Grishakov, P.N. Degtyarenko, N.N. Degtyarenko, V.F. Elesin, V.S. Kruglov. Russ.Phys. J. 52, 11 (2009).

[9] K.S. Grishakov, P.N. Degtyarenko, N.N. Degtyarenko, V.F. Elesin, V.S. Kruglov. Phys. Proc. 36 (2012).

[10] П.И. Безотосный, С.Ю. Гаврилкин, А.Н. Лыков, А.Ю. Цветков. Кр. сообщения по физике ФИАН 6, 3 (2014).

[11] П.И. Безотосный, С.Ю. Гаврилкин, А.Н. Лыков, А.Ю. Цветков. Кр. сообщения по физике ФИАН 12, 26 (2014).

[12] П.И. Безотосный, С.Ю. Гаврилкин, А.Н. Лыков, А.Ю. Цветков. ФТТ 57, 7, 1277 (2015).

[13] П.И. Безотосный, С.Ю. Гаврилкин, К.А. Дмитриева, А.Н. Лыков, А.Ю. Цветков. ФТТ 61, 2, 234 (2019).

[14] П.И. Безотосный, С.Ю. Гаврилкин, К.А. Дмитриева, А.Н. Лыков, А.Ю. Цветков. Кр. сообщения по физике ФИАН 2, 20 (2020).

[15] P.I. Bezotosnyi, K.A. Dmitrieva, S.Yu. Gavrilkin, A.N. Lykov, A.Yu. Tsvetkov. IEEE Transact. Appl. Supercond 31, 3, 7500107 (2021).

[16] П.И. Безотосный, К.А. Дмитриева. ФТТ 63, 8, 1035 (2021).

[17] С.Ю. Гаврилкин, А.Н. Лыков, А.Ю. Цветков, П.И. Безотосный. Кр. сообщения по физике ФИАН 2, 29 (2018).

[18] А.Н. Лыков, А.Ю. Цветков, Г.Ф. Жарков. ЖЭТФ 128, 392 (2005).

[19] A.N. Lykov, A.Yu. Tsvetkov. Phys. Rev. B 76, 14, 144517 (2007).

Редактор Т.Н. Василевская 